\title{
Model-Predictive-Control for Dual-Active-Bridge Converters Supplying Pulsed Power Loads in Naval DC Micro-grids
}

\author{
Linglin Chen, Shuai Shao, Member, IEEE, Qian Xiao, Luca Tarisciotti, Member, IEEE, \\ Patrick W. Wheeler, Senior Member, IEEE, and Tomislav Dragicevic, Senior Member, IEEE
}

\begin{abstract}
Pulsed-Power-Loads (PPLs) are becoming prevalent in medium-voltage naval DC micro-grids. To alleviate their effects on the system, energy storages are commonly installed. For optimal performance, their interface converters need to have fast dynamics and excellent disturbance rejection capability. Moreover, these converters often need to have voltage transformation and galvanic isolation capability since common energy storage technologies like batteries and super-caps are typically assembled with low voltage strings. In order to address these issues, a Moving-Discretized-Control-Set Model-PredictiveControl (MDCS-MPC) is proposed in this paper and applied on a Dual-Active-Bridge converter. Fixed switching frequency is maintained, enabling easy passive components design. The proposed MDCS-MPC has a reduced prediction horizon, which allows low computational burden. The operating principle of the MDCS-MPC is introduced in development of a cost function that provides stiff voltage regulation. Resonance damping and sampling noise resistance can also be achieved with the proposed cost function. An adaptive step is introduced to enable fast transition. Assessments on the performance of the proposed MDCS-MPC are conducted. Comparisons with other control methods are also provided. Experimental validations on a $300 \mathrm{~V} / 300 \mathrm{~V} 20 \mathrm{kHz} 1 \mathrm{~kW}$ Dual-Active-Bridge converter are carried out to verify the theoretical claims.
\end{abstract}

Index Terms- Isolated DC/DC converter, Dual-Active-Bridge (DAB), Model Predictive Control (MPC).

\section{INTRODUCTION}

The early electrification attempt on a small electric propelled boat dates back to late 1830s [1]. Since then, there has been a great evolution of the technologies developed for marine vessels [2]. After more than 100 years of research on shipboard electrical power system, with the recent advancement in power electronics, DC on-board micro-grids

Manuscript received March 25, 2019; accepted May 14, 2019. This work was supported in part by the Office of Naval Research Global under the award number N62909-17-1-2106. (Corresponding authors: Shuai Shao, Qian Xiao)

L. Chen and P. Wheeler are with the Department of Electrical and Electronics Engineering, University of Nottingham, Nottingham, U.K. Email: (eexlc15; eezpww)@nottingham.ac.uk

S. Shao is with the College of Electrical Engineering, Zhejiang University, Hangzhou 310027, China. E-mail: shaos@zju.edu.cn

Q. Xiao is with the Key Laboratory of Smart Grid of Ministry of Education, Tianjin University, China. E-mail: xiaoqian@tju.edu.cn

L. Tarisciotti is with the Department of Engineering, Universidad Andres Bello, Santiago, Chile. E-mail: luca.tarisciotti@unab.cl

T. Dragičević is with the Department of Energy Technology, Aalborg University, 9220 Aalborg East, Denmark. E-mail: tdr@, et.aau.dk becomes a prevailing choice for naval vessels [3]-[6].

In a typical shipboard power system shown in Fig. 1, there exists some particular loads like radars, sonars and electromagnetics weapons which intermittently draw a large amount of power from the power system [7]. Collectively, they are referred to as Pulse-Power-Loads (PPLs). A dual $\mathrm{DC} / \mathrm{DC}$ converter structure is usually preferred as the direct interface supplying high power PPLs [8]. The dual DC/DC provides mainly reactive power required by PPLs while the active power is soured from the DC bus. The existence of the PPLs poses potential danger for the operation of the naval onboard DC micro-grids. The behavior of repeatedly large variation of load current presents challenges in DC bus voltage regulation. DC bus voltage sag and overshoot may cause shutdown of propulsion system, combat weapons and other sensitive loads. Therefore, dedicated Energy Storage Systems (ESS) have been usually integrated to coexist with PPLs in the micro-grids through the interface of Dual-Active-Bridge (DAB) converters [6]. Admittedly, DAB topology and its relevant derivatives [9], [10] have drawn considerable attention in the application of DC micro-grids for their salient merits in high frequency galvanic isolation, high voltage step up/down ability and high power conversion efficiency [11]. Apart from that, dynamic performance of the interface converters is also instrumental for powering the PPLs, and this is largely dependent on the control algorithm applied to the interface converters.

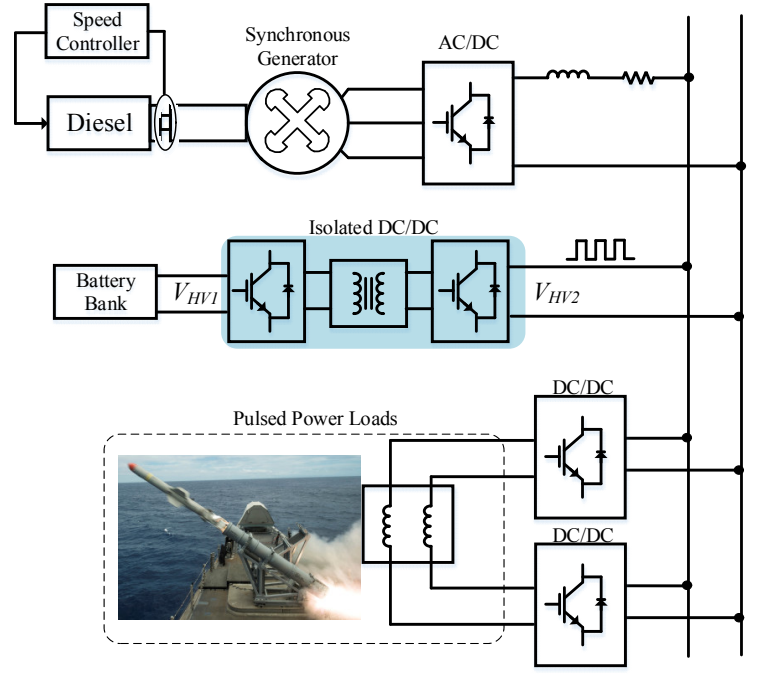

Fig. 1. A simplified diagram of the naval shipboard DC micro-grids. 


\section{IEEE POWER ELECTRONICS REGULAR PAPER}

Predictive control is often considered in power electronics converters for several advantages it can provide, such as fast dynamics, easy inclusion of constraints, and simple digital implementation. In particular, Finite-Control-Set ModelPredictive-Control (FCS-MPC) has been investigated in AC power conversion [12]-[14]. However, the application of predictive control in DC/DC converters has not been so intensively explored; FCS-MPC methods proposed for use in the boost converter with receding horizon by $P$. Karamanakos et al. [15] and B. Wang et al. [16] demonstrated fast dynamics. However, these approaches resulted in variable switching frequency and demanded heavy computation. F. M. Oettmeier et al. [17] proposed a Continuous-Control-Set ModelPredictive-Control (CCS-MPC) also for boost converters which effectively avoided voltage transition overshoot. Above approaches [15]-[17] are not applicable in DAB converters in presence of high frequency transformer.

P. Akter et al. [18] presented a model predictive control for the bidirectional isolated DC-DC converters which were operated at $100 \mathrm{kHz}$ switching frequency with only $4 \mathrm{kHz}$ nominal transformer current. This fell into the category of application of MPC in AC power conversions [12] where switching frequency was much higher than fundamental current. In this paper, the control approach is investigated for DAB converters operating with a transformer current frequency close to the switching devices.

A dead beat control was proposed for DAB converters by S. Dutta et al. [19], [20]. They have used high bandwidth current sensors to accurately sample instant high frequency transformer current, therefore fast transition was obtained. Semi-predictive approaches were also investigated by researchers. Z. Shan et al. [21] proposed a method with feedforward loop to improve the dynamic performance. The feedforward loop shared similarity to [19] with respect to the requirement in instant high frequency current sampling. Above methods [19]-[21] have limitations in DAB converters operating in high power/high switching frequency as the sampling of the instant transformer current becomes more challenging. To the best knowledge of authors, no mature product are available in the market for current sensing with bandwidth above $2 \mathrm{MHz}$ and current rating higher than $100 \mathrm{~A}$.

A virtual direct power control scheme for DAB converters that prescribed less for current sensors was proposed by $W$. Song et al. [22]. This method calculated the control output from the expression of power. However, this method still relies on the PI controller and demonstrated limited load current disturbance rejection.

A Moving-Discretized-Control-Set Model-PredictiveControl (MDCS-MPC) is proposed in this paper with the aim of controlling the voltage on the PPLs. MDCS-MPC requires low bandwidth current sensing compared to [19]-[21]. Therefore it is potentially more suitable for high power/high frequency $\mathrm{DAB}$ converters. Performance merits are summarized as follows:

1. Low computational burden. The proposed MDCS-MPC demands much less computational power compared to the approach introduced in [15]. MDCS-MPC is implemented in a commercial control platform TMS320F2837xD in this paper.

2. Fixed switching frequency. This eases passive components design;

3. Fast dynamics. An adaptive step has been implemented in MDCS-MPC for fast transition;

4. Voltage deviation suppression. A term with the voltage slope constraint is proposed in the cost function. It can achieve resonance damping and increase resiliency to sampling noise.

This paper is organised as follows: in Section II, the proposed MDCS-MPC is introduced. In Section III, performance of the MDCS-MPC is evaluated and compared with other existing control methods for DAB converters. Finally, experimental results are presented for a $300 \mathrm{~V} / 300 \mathrm{~V}$ $20 \mathrm{kHz} 1 \mathrm{~kW}$ DAB converter in Section V.

\section{PROPOSED MDCS-MPC}

The schematic of the DAB converter is shown in Fig. 2. $\mathrm{H}$-bridges at each side of the high frequency transformer generate voltages $v_{a c 1}$ and $v_{a c 2}$, as shown in Fig. 3. Voltages $v_{a c 1}$ and $v_{a c 2}$ have a fundamental frequency of $f_{s}$. They are exerted on power transferring inductor $L_{p}$, producing transformer current $i_{a c 1}$. $T_{s}$ denotes one switching period. The phase shift value $\left(D_{\varphi} T_{s}\right)$ between $v_{a c 1}$ and $v_{a c 2}$ is controlled to transfer the power from the DC bus to PPLs.

Various modelling methods such as reduced order model [23], [24], improved reduced order [25], generalized average model[26] and discrete time model [27] have been proposed for DAB and the reduced order model [23], [24], presented in Fig. 4 and (1) has a good compromise in complexity and accuracy, and are utilized in this work. Prediction error due to inaccurate model may occur, however, like in all other model predictive control, tracking error compensation can be implemented [19], [28]. A proportional prediction error compensation loop has been implemented to address this issue. This will be discussed later in Section II.C.

$$
<i_{H V 2}>_{T s}=\frac{V_{H V 1}}{f_{s} L_{p}} D_{\varphi}\left(1-2 D_{\varphi}\right)
$$

In this paper, we assume the scenario where ESS is the only available power source in the system. The main objective of the designed control in this case is to regulate voltage $V_{H V 2}$ suppling PPLs. The dynamic equation of the output voltage is developed as follows:

$$
C_{H V 2} \frac{d<V_{H V 2}>_{T s}}{d t}=<i_{H V 2}>_{T s}-<I_{\text {load }}>_{T s}
$$

Discretizing (2) using forward approximation yields:

$$
V_{H V 2}[k+1]=\frac{i_{H V 2}[k]-I_{\text {load }}[\mathrm{k}]}{C_{H V 2} f_{s}}+V_{H V 2}[k]
$$

Assuming load current does not vary drastically in one sampling period:

$$
I_{\text {load }}[k]=I_{\text {load }}[k+1]
$$

The prediction for output voltage at time instance $k+2$ is:

$V_{H V 2}[k+2]=\frac{i_{H V 2}[k+1]-I_{\text {load }}[\mathrm{k}]}{C_{H V 2} f_{s}}+V_{H V_{2}}[k+1]$ 
Substituting $V_{H V 2}[k+1]$ in (3) to (5), yields

$V_{H V^{2}}[k+2]=V_{H V^{2}}[k]$

$$
+\frac{i_{H V 2}[k+1]+i_{H V 2}[k]-2 I_{\text {load }}[k]}{C_{H V 2} f_{s}}
$$

, where $i_{H V 2}[k+1]$ and $i_{H V 2}[k]$ can be easily derived from (1).

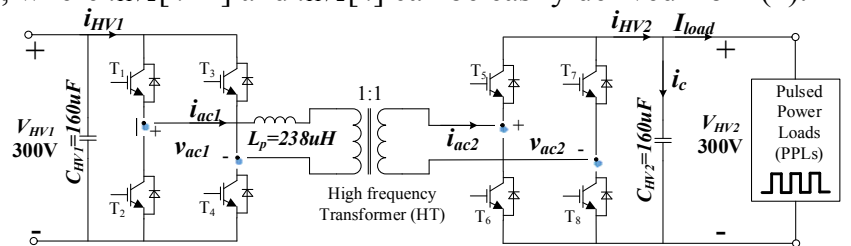

Fig. 2. The schematic of a Dual-Active-Bridge (DAB) converter.

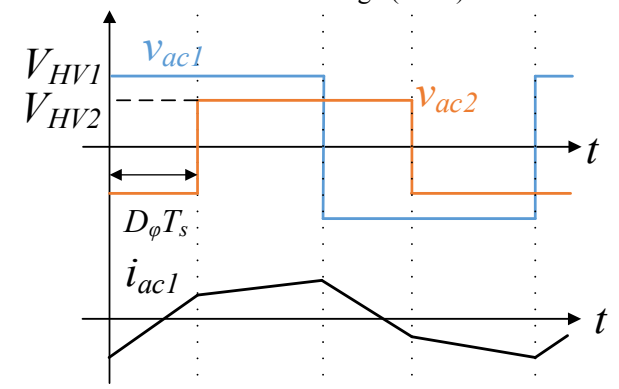

Fig. 3. Conceptual transformer voltage and current waveforms

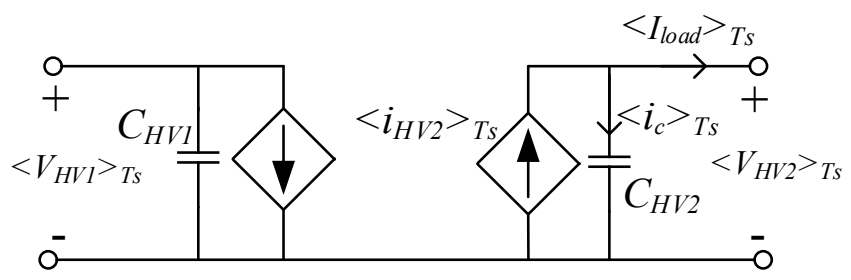

Fig. 4. The averaged model of a DAB converter [23], [24].

\section{A. The operating principle}

The proposed MDCS-MPC controls the converter output voltage $V_{H V 2}$ based on the discretized average model of the DAB in (6). A preliminary cost function is proposed as in (7) with the only purpose of regulating voltage $V_{H V 2}$ to reference $V_{H V 2}$ ref. It is worth mentioning that (7) is not the finalized cost function, but a simple one meant to help illustrate the operating principle of the proposed MDCS-MPC.

$$
c t=\left(V_{H V 2 \_r e f}-V_{H V 2}[k+1]\right)^{2}
$$

The variable $D_{\varphi}$ in (1) is continuous in nature. However, in digital control, $D_{\varphi}$ needs to be discretized. The discretization precision is subjected to the control platform applied. $\Delta_{f}$ is defined as the finest phase shift value that can be achieved in a digital control platform. For unidirectional power flow, DAB works predominately in the range:

$$
D_{\varphi} \in[0,0.5]
$$

(8) is further discretized into $\mu_{m}\left(=0.5 / \Delta_{f}+1\right)$ elements as described in (9).

$$
D_{\varphi} \in\left\{0, \Delta_{f}, 2 \Delta_{f}, \cdots, 0.5\right\}
$$

In order to realize a control algorithm that is feasible on standard commercial microcontrollers, the proposed MDCSMPC evaluates a reduced number of values in each sampling period. In one sampling period, $\mu\left(\mu \leq \mu_{m}\right)$ number of points are assessed. They are centered at the previous working point.

An intuitive mechanism illustration of the proposed MDCS-MPC is depicted in Fig. 5. In the control interval $k$ to $k+1, \mu=3$ points are evaluated centred at the previous working point a. The current Discretized-Control-Set (DCS) is $\left\{a-\Delta_{f}, a\right.$, $a+\Delta f\}$. Value $a+\Delta f$ results in the smallest cost function $(c t)$, therefore, apply this value at time instance $k+1$. In the next control interval $k+1$ to $k+2$, the same process is repeated, and the DCS moves to $\left\{a, a+\Delta_{f}, a+2 \Delta_{f}\right\}$. The DCS is moving with the working point in the domain as (9). In this control interval, value $a+2 \Delta_{f}$ results in the smallest cost function (ct), therefore, this value is applied at the time instance $k+2$. This process goes on.

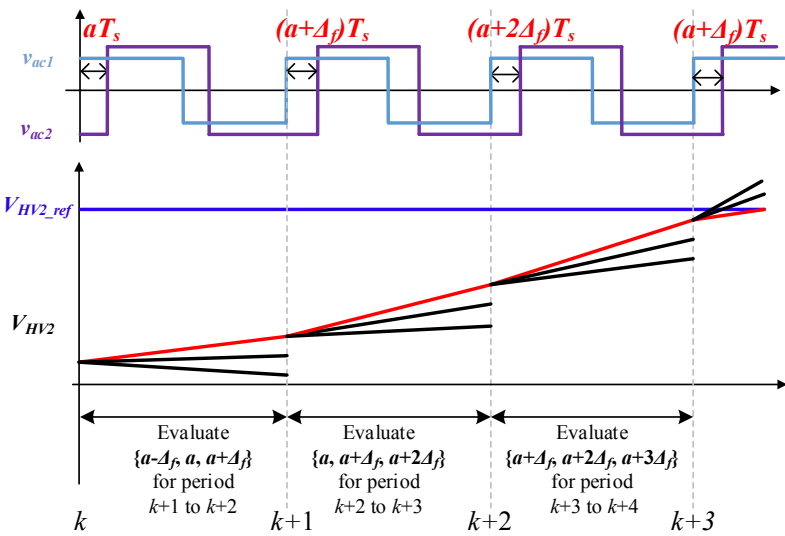

Fig. 5. The operating principle of the proposed MDCS-MPC for DAB. $\mu$ is set to be 3 in this illustration.

\section{$B$. The proposed cost function \& adaptive step}

Taking into account the computational delay, MDCSMPC has a prediction horizon of two sampling periods. Therefore, the cost function is proposed as follows:

$$
c t=\alpha_{1} G_{1}+\alpha_{2} G_{2}
$$

where,

$$
\left\{\begin{array}{l}
G_{1}=\left(V_{H V 2}{ }_{r e f}-V_{H V 2}[k+2]\right)^{2} \\
G_{2}=\left(V_{H V 2}[k+2]-V_{H V 2}[k]\right)^{2}
\end{array}\right.
$$

The first term $G_{1}$ is responsible for regulation of the output voltage $V_{H V 2}$ to reference value $V_{H V 2}$ ref while the second term $G_{2}$ takes charge of voltage deviation reduction. This can thus achieve resonance damping and resistance enhancement to sampling noise. When $V_{H V 2}$ is far from the reference value, $G_{l}$ plays a dominant role in the cost function. However, when $V_{H V 2}$ reaches close to $V_{H V 2}$ ref, $G_{2}$ starts to take effect. $G_{2}$ limits the variation of $V_{H V}$. This essentially prevents $V_{H V 2}$ from dithering due to analogue to digital sampling noise. $G_{2}$ also alleviates the oscillation during load transition. Tuning of weighting factors $\alpha_{1}$ and $\alpha_{2}$ is crucial to the performance of the proposed controller. T. Dragicevic et al. [29] proposed an artificial neural network approach to best tune the weighting factors in MPC. This approach is also used in this paper.

Larger values of $\mu$ can increase the transition dynamics, but it aggravates the computational burden to the real-time 
digital controller. Therefore, an adaptive step is adopted instead of the finest search step $\Delta f$. Define the adaptive step as:

$$
\begin{gathered}
V_{\Delta}=\left\{\begin{array}{c}
\left|V_{H V 2_{-} r e f}-V_{H V 2}[k]\right|,\left|V_{H V 2_{-} r e f}-V_{H V 2}[k]\right|<V_{m} \\
V_{m},\left|V_{H V 2_{-} r e f}-V_{H V 2}[k]\right|>V_{m}
\end{array}\right. \\
\Delta_{a d p}=\Delta_{f}\left(1+\lambda V_{\Delta}\right)
\end{gathered}
$$

where $V_{m}$ is the saturated voltage. $\lambda$ is a coefficient determined according to the requirement of transition performance. $\lambda$ and $V_{m}$ are set as 1 and $20 \mathrm{~V}$ respectively in the following simulation and experiment validations.

A diagram of the calculation of the adaptive step is depicted in Fig. 6. The adaptive step $\Delta_{a d p}$ changes with the deviation of the output voltage to the reference. When $V_{H V 2}$ is far from the reference, $\Delta a d p$ grows large. In contrast, when $V_{H V 2}$ equals to the reference, $\Delta a d p$ becomes $\Delta f$. Such that, the control accuracy remains.

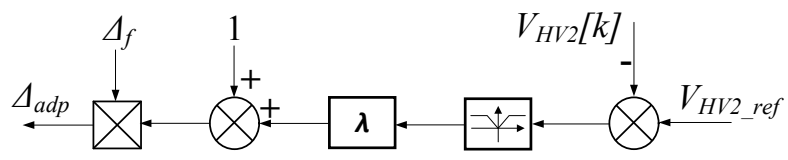

Fig. 6. A diagram of the adaptive step.

\section{The compensation loop for error corrections}

There are generally two ways of improving the prediction accuracy. The first approach is to use a more precise mathematical model of the DAB converter as investigated [25], [30]. However, those high order models increase the computational burden. The second resort involves the feedback compensation. Methods commonly seen in other predictive controls can be well suited in the proposed MDCSMPC [19], [28], [31], [32]. This paper presented a method similar to the one proposed by K. Shen et al. [28].

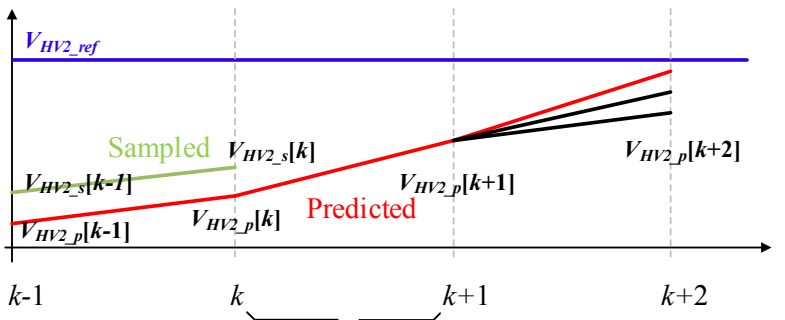

ct evaluation with corrected prediction $V_{H V 2_{-}}[k+2]$

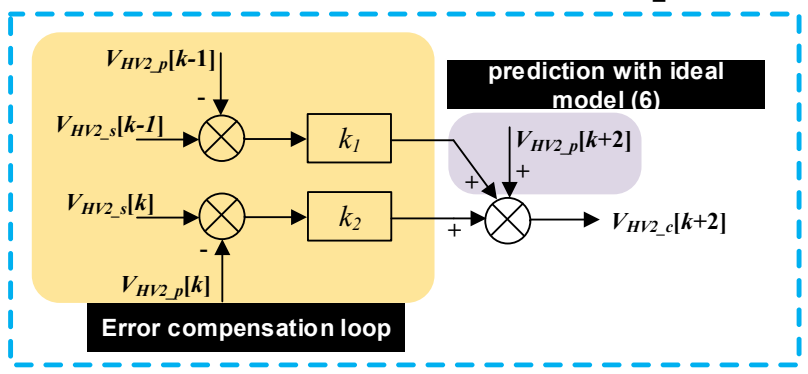

Fig. 7. Prediction error compensation loop.

Additional compensation loop to correct the modeling error is depicted in Fig. 7. $V_{H V 2} p$ is the predicted output voltage $\left(V_{H V 2}\right)$ value using the ideal DAB model in (6). $V_{H V 2}[\mathrm{k}+2]$ is the voltage prediction during control period $k$ to $k+1$ with MDCS. $V_{H V 2} s$ is the sampling voltage value. The voltage error compensation value is the weighted sum of the error between voltage sampling $V_{H V 2_{-} s}$ and prediction $V_{H V 2} p$ values in previous two steps.

It is worth mentioning that parameters $k_{1}$ and $k_{2}$ can be designed to ensure the Lyapunov stability [32]. However, this paper focused on the MDCS-MPC concept, the impact study of $k_{1}$ and $k_{2}$ has not been carried out. Weighting factors $k_{1}$ and $k_{2}$ are tuned empirically here as 0.5 and 0.25 , respectively.

\section{The flow chat of MDCS-MPC}

The flow chat of the proposed MDCS-MPC is presented in Fig. 8. It shows the calculation process of MDCS-MPC in the control period $k$ to $k+1$. The output of the control is the optimal control variable in MDCS to be applied at time instance $k+2 . \mu(=3)$ elements are utilized in the MDCS for illustration purpose. It can be expanded on request of the particular applications.

The evaluation process starts with the calculation of the adaptive step $\Delta_{a d p}(12)$. It determines the step distance between two near values in MDCS. MDCS slides in the range described in (8) centered at the control value $D_{\varphi}[k+1]$ which is going to be applied at time instance $k+1$. Then the iteration starts for MDCS-MPC. The bus voltage is predicted ( $V_{H V 2} p$ ) with three elements in MDCS. Each voltage prediction is corrected by the compensation loop illustrated in Fig. 7. Finally, cost functions (10) are calculated with each corrected voltage prediction value $\left(V_{H V 2} \_\right.$) for comparison of minimal. The element achieving minimal $c t$ is then stored in $D_{\varphi}[k+2]$ which is going to be applied at time instance $k+2$.

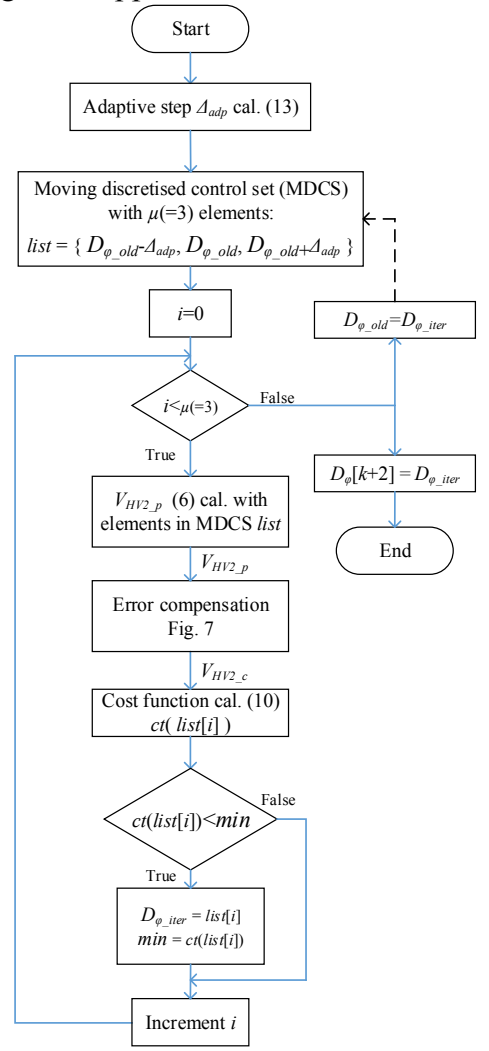

Fig. 8. The flow chat of the proposed MDCS-MPC. 


\section{IEEE POWER ELECTRONICS REGULAR PAPER}

\section{PERFORMANCE EVALUATIONS}

Evaluations for the proposed MDCS-MPC controller have been carried out in this section in both time and frequency domain using simulation software PLECS/Simulink. Controllers demanding the same sampling requirement are considered for fair comparisons. The proposed methodology has been compared with a "PI controller" [33], "hybrid phase shift control" [24] and a "Virtual Direct Power Control (VDPC) scheme" [22]. The specification of the DAB converter under evaluation is listed in Table I. TABLE I

CONVERTER PARAMETERS

\begin{tabular}{|l|c|c|}
\hline Description & Value & Units \\
\hline Switching frequency $f_{s}$ & 20 & $\mathrm{kHz}$ \\
\hline Dead time $t_{d}$ & 2.5 & $\mu \mathrm{S}$ \\
\hline Transformer turn ratio & $20: 20$ & $/$ \\
\hline Primary power inductor $L_{s}$ & 283 & $\mu \mathrm{H}$ \\
\hline Primary DC capacitor $C_{H V 1}$ & 160 & $\mu \mathrm{F}$ \\
\hline Secondary DC capacitor $C_{H V 2}$ & 160 & $\mu \mathrm{F}$ \\
\hline Rated power & 1 & $\mathrm{~kW}$ \\
\hline
\end{tabular}

\section{A. Load disturbance rejection}

Output impedance is selected as the metric of evaluating the ability of load current disturbance rejection. In order to assess the impedance of a converter, the small signal model of $\mathrm{DAB}$ converters is often carried out by neglecting the dynamic on $i_{a c 1}$ [34], but this is insufficient to describe the DAB converters especially when power inductance $L_{p}$ is comparatively large as with the DAB under test in this paper. T. Dragicevic introduced a describing function approach [35]. This approach is well suited for evaluating impedance of DAB converters even with non-linear controllers.

An output impedance evaluation circuit diagram is implemented in the PLECS/Simulink software as shown in Fig. 9. The DAB converter has been simplified as a voltage source $V_{\text {out }}$ and an output impedance $Z_{\text {out }}$. $I_{d c}$ represents the full power steady-state load current which sets the equilibrium point. $i_{a c}$ stands for the injected small current which provides small signal perturbation. The output voltage $V_{H V 2}$ of the DAB converter is measured at each frequency of the injected current $i_{a c}$. Therefore, the output impedance can be calculated as:

$$
Z_{\text {out }}(f)=\frac{V_{H V 2}(f)}{i_{\text {ac }}(f)}
$$

\section{DAB converter}

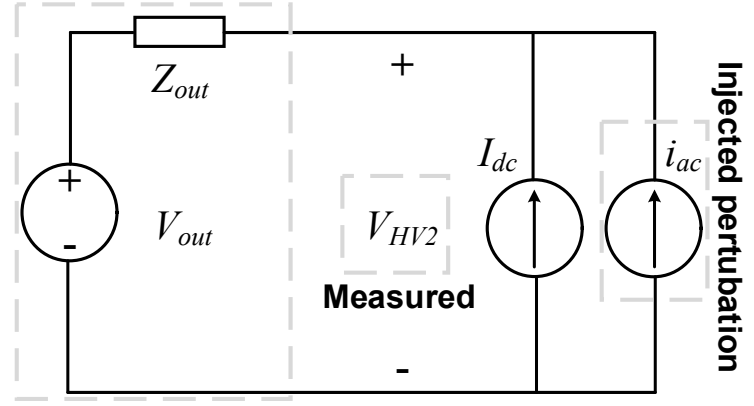

Fig. 9. The output impedance evaluation circuit.

The output impedance of using the proposed MDCSMPC, PI, hybrid controller and VDPC controllers are illustrated in Fig. 10. It is easy to observe from the figure that MDCS-MPC can maintain low output impedance for the DAB converter in a wide frequency range until it reaches $0 \mathrm{~dB}$ at 1 $\mathrm{kHz}$. In contrast, when the PI controller and VDPC are used, output impedances rise above $0 \mathrm{~dB}$ at $40 \mathrm{~Hz}$ and $80 \mathrm{~Hz}$, respectively. The hybrid controller showed similar trend in $Z_{\text {out }}$ as with MDCS-MPC for frequency range $10 \mathrm{~Hz}$ to $210 \mathrm{~Hz}$. However, $Z_{\text {out }}$ increases drastically to $0 \mathrm{db}$ between $210 \mathrm{~Hz}$ to $350 \mathrm{~Hz}$. Conclusively, MDCS-MPC shows smallest $Z_{\text {out }}$ below $500 \mathrm{~Hz}$ when compared to PI, hybrid controller and VDPC scheme. Therefore, MDCS-MPC has greater load current disturbance rejection ability.

Simulation in time domain has also been carried out confirming the conclusion made above as shown in Fig. 11. The PPL current poses abundant harmonics/disturbance to the DAB converter. The output voltage $V_{H V 2}$ is measured in the time domain when the DAB is loaded with pulsed current $I_{\text {load }}$. The result is consistent with the conclusion made from the frequency domain, load voltage $V_{H V 2}$ demonstrated descending oscillation and variation when PI, VDPC, hybrid controller and MDCS-MPC are applied subsequently.

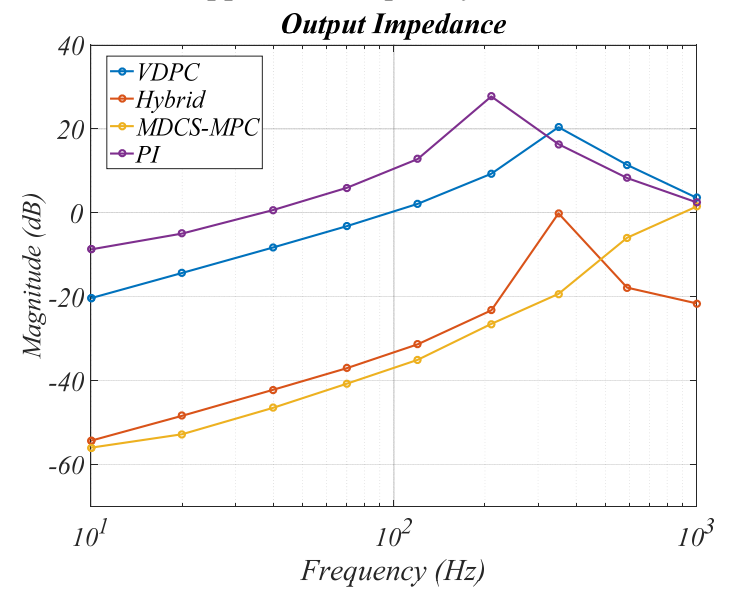

Fig. 10. Output impedance comparisons for PI [33], VDPC scheme [22], hybrid controller [24] and proposed MDCS-MPC.

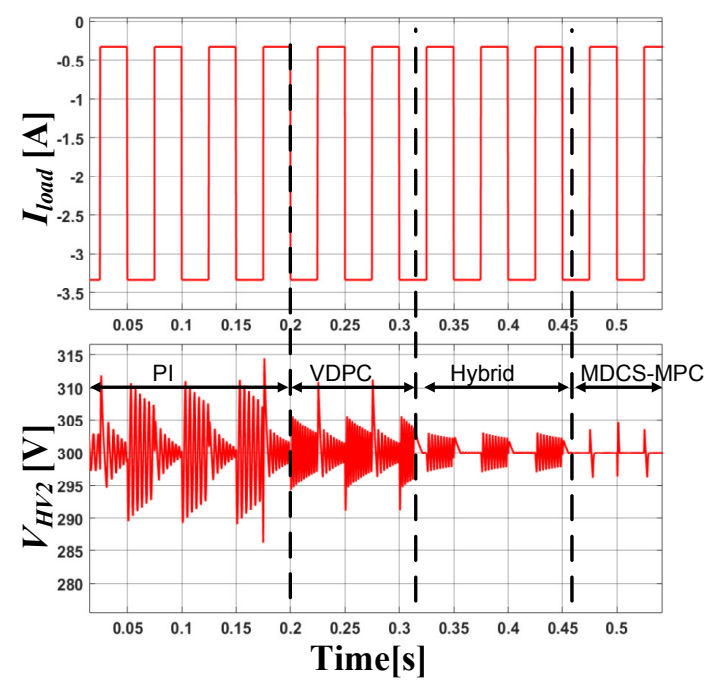

Fig. 11. Time domain load disturbance rejection ability comparison. 


\section{B. Source voltage disturbance rejection}

Simulations have also been conducted for evaluating source voltage disturbance rejection. An evaluation circuit diagram is implemented in the PLECS/Simulink software as shown in Fig. 12. The source voltage can be decomposed by DC component $V_{d c}$ and AC components $V_{a c}$. $I_{d c}$ and $V_{d c}$ set the equilibrium point. $v_{a c}$ corresponds to the injected voltage which provides small signal perturbation. The output voltage $V_{H V 2}$ of the DAB converter is measured respectively for each injected frequency. Therefore, the source voltage disturbance rejection metric $G_{v}$ is defined as:

$$
G_{v}(f)=\frac{V_{H V 2}(f)}{v_{a c}(f)}
$$

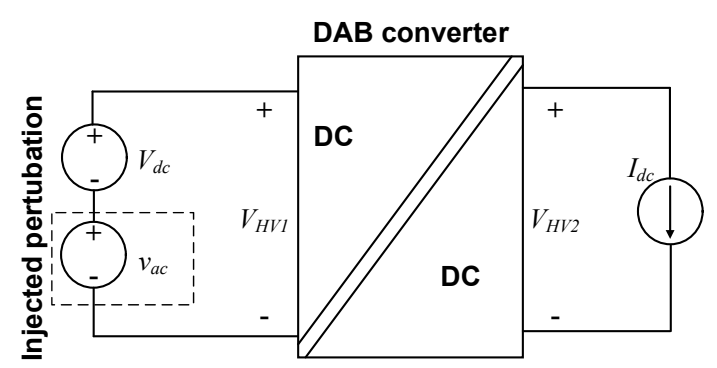

Fig. 12. The circuit to emulate the voltage drop on supercaps with PPLs.

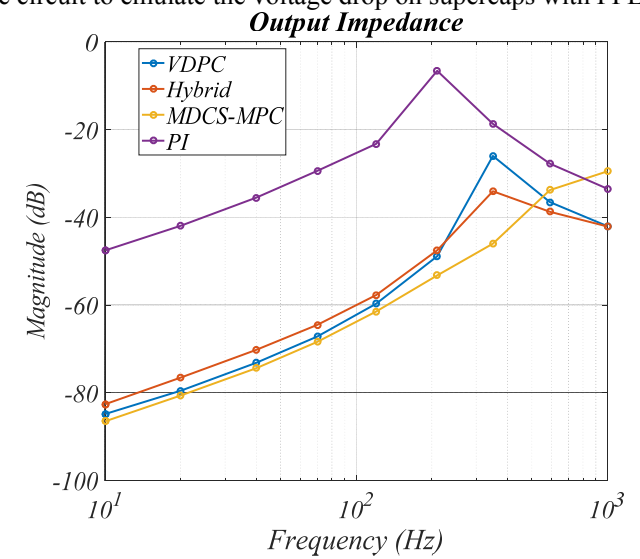

Fig. 13. $G_{V}$ comparisons for PI [33], VDPC scheme [22], hybrid controller [24] and proposed MDCS-MPC.

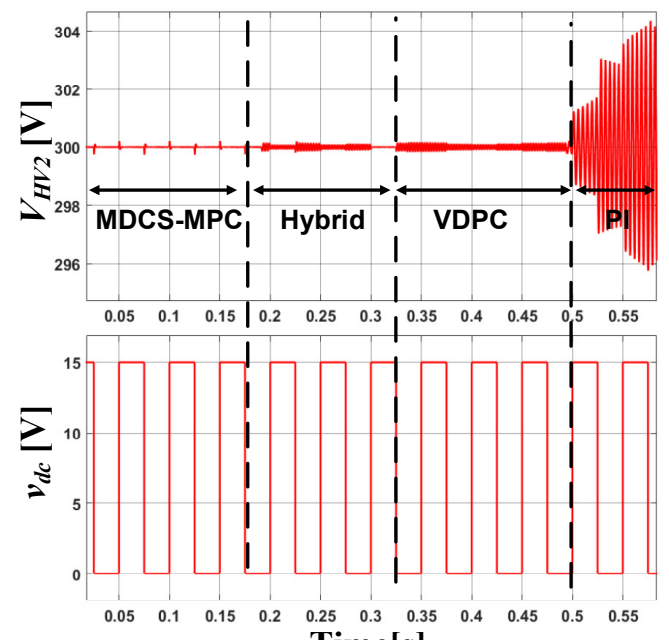

Time[s]

Fig. 14. Time domain source voltage disturbance rejection ability comparison.
$G_{v}$ of using the PI, VDPC, hybrid controller and proposed MDCS-MPC are illustrated in Fig. 13. When the PI controller is used, $G_{v}$ rises from $-48 \mathrm{~dB}$ to $-8 \mathrm{~dB}$ in the range from $10 \mathrm{~Hz}$ to $200 \mathrm{~Hz}$. The hybrid controller shows similar performance with the VDPC scheme. $G_{v}$ of using VDPC and hybrid controller are well below the one with PI controller. MDCS-MPC shows the smallest amplitude of $G_{v}$ in the low frequency range, therefore it holds the best performance for source voltage disturbance rejection among all.

Above conclusion has also been confirmed with the simulation results in Fig. 14. A $50 \%$ duty cycle, $15 \mathrm{~V}$ pulse voltage $v_{a c}$ has been placed in series with $300 \mathrm{~V} V_{d c}$. The largest variation in $V_{H V 2}$ is $0.2 \mathrm{~V}$ when using MDCS-MPC, VDPC and hybrid controller. The difference is that the voltage waveform with MDCS-MPC presents least oscillation. In contrast, when PI controller is utilized. Large variation and oscillation in $V_{H V 2}$ occurs.

\section{Remarks on the evaluated controllers}

According to the evaluations in load/source disturbance rejection abilities, MDCS-MPC shows the best performance. The hybrid controller ranks the second, then comes the VDPC, and PI controller shows the least capability for both disturbance rejections. However, the hybrid controller and VDPC demonstrated issues in the practical implementation described as follows;

1) The hybrid controller requires load estimation. H. Bai [24] assumed resistive loads for the DAB converter. However, in the naval DC micro-grid, load impedance is not always resistive especially when long power cable and power electronics converters on the DC bus are taken into account. The estimation of load could be erroneous.

2) The VDPC scheme demonstrated issues at low output current and low output voltage. The PI gain has to be adaptable with the output current, otherwise instability could happen at light load.

In conclusion, although VDPC and hybrid controls demonstrated better performance compared to the PI controller in simulations, they are not considered in practice due to the major issues described above. In the following experiment section, comparisons are only carried out between MDCSMPC and the PI controller.

\section{EXPERIMENT}

The proposed methodology has been validated on a $1 \mathrm{~kW}$ $20 \mathrm{kHz} 300 \mathrm{~V} / 300 \mathrm{~V}$ laboratory prototype. The experiment test circuit diagram is depicted in Fig. 15 where the PPLs are emulated by a solid-state circuit breaker (SSCB) and resistive loads $R$. When $\mathrm{S}$ is on, bench power supply EA-PS 9360-40 $3 \mathrm{U}$ (1 Output, $0 \mathrm{~V}-360 \mathrm{~V}, 0 \mathrm{~A}-40 \mathrm{~A}$ ) is connected directly to the DAB providing stiff input voltage $V_{s}$. When $\mathrm{S}$ is off, an inductor $L_{s r c}(1 \mathrm{mH})$ is inserted in series with the power supply. $L_{s r c}$ could represent long cable inductance. $L_{s r c}$ will cause $V_{H V I}$ voltage variation when the DAB is loaded with PPLs.

The experiment prototype is shown in Fig. 16. A TMS320F2837xD evaluation board from Texas Instruments has been adopted as the digital control platform which communicates with a host computer. $\mu$ is set to 7 , and the 


\section{IEEE POWER ELECTRONICS REGULAR PAPER}

weighting factors are set as $\alpha_{l}=1$ and $\alpha_{2}=5$ in the experiment, otherwise specified. The main components used in the prototype are listed in Table II.

The computation time of the proposed MDCS-MPC is evaluated as shown in Fig. 17. The PI controller takes 1.16us to run while, in contrast, the time to run MDCS-MPC varies with $\mu$. In the experiment, $\mu=7$ has already demonstrated good performance against PI controller, and it only takes 7.8us. Since $20 \mathrm{kHz}$ switching frequency is utilized, $50 \mathrm{us}$ is available in one sampling period. Therefore, there is sufficient headroom for implementing A/D sampling, digital filters, MODBUS communication, protections etc. TABLE II

\begin{tabular}{|c|c|c|}
\multicolumn{3}{c}{ HARDWARE COMPONENTS } \\
\hline Component & Description & Parameters \\
\hline Switching devices & SKM75GB128D & $\begin{array}{c}V_{C E S}=1200 \mathrm{~V} ; \\
I_{c}=100 \mathrm{~A}\end{array}$ \\
\hline Pri/Sec capacitor bank & $1848 \mathrm{~S}$ MKP & $700 \mathrm{~V} ; 20 \mathrm{uF} * 8$ \\
\hline Magnetic components & Ferrites; litz wire & $\begin{array}{c}L_{m}=3 \mathrm{mH} ; \\
L_{p}=0.28 \mathrm{mH}\end{array}$ \\
\hline Voltage sensors & LV 25-P & $t_{r}=40 \mathrm{us}$ \\
\hline Current sensors & LA $55-\mathrm{p}$ & $\begin{array}{c}\text { BW(-1dB) } \\
200 \mathrm{kHz}\end{array}$ \\
\hline
\end{tabular}

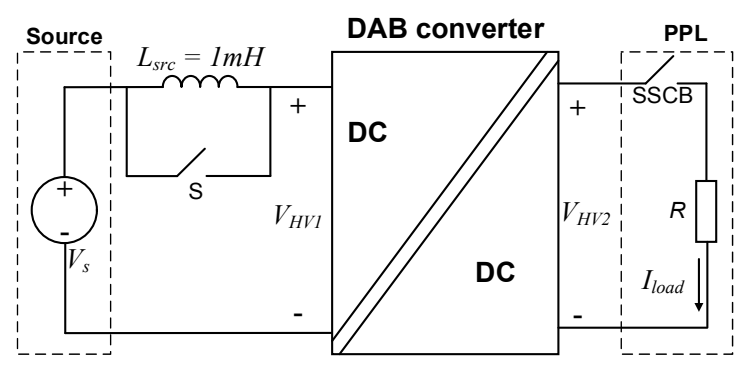

Fig. 15. The experiment test circuit diagram.

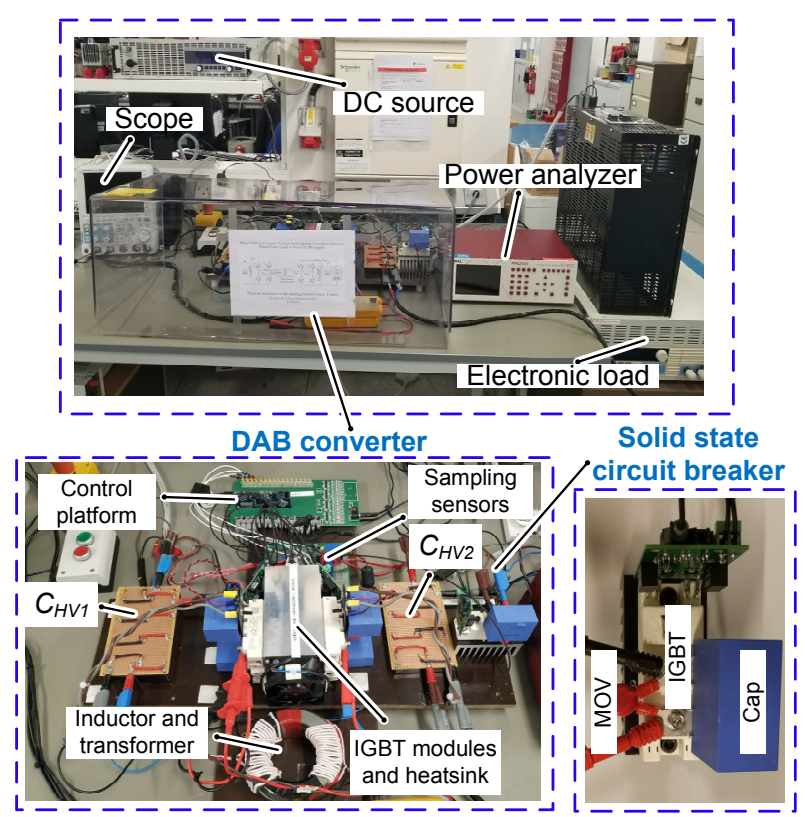

Fig. 16. The experiment setup.

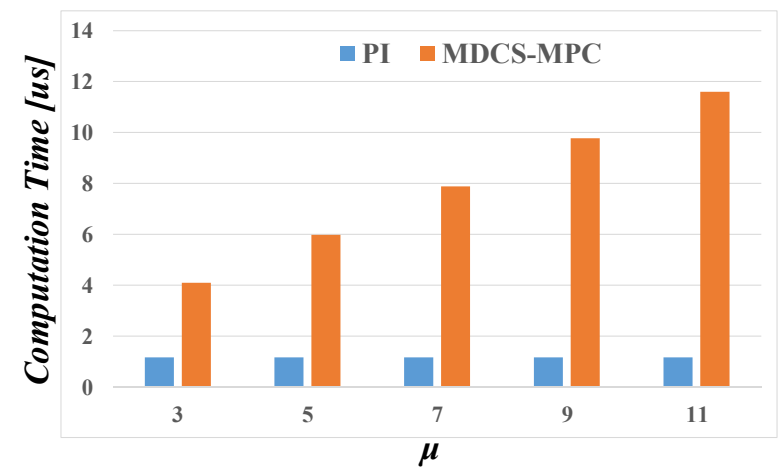

Fig. 17. Computation time comparison between MDCS-MPC and PI control.

The effectiveness of $G_{2}$ and adaptive step has been verified in the experiment. Results are shown in Fig. 18 and Fig. 19. Enhancement on sampling noise resistance is proofed by Fig. 18. It is clearly shown that the steady state dithering is alleviated when $G_{2}$ is enabled. The acoustic noise from transformer has also been alleviated. The comparison between Fig. 19 (a)(b) and (c)(d) verifies the resonance damping effect with $G_{2}$. The comparison between Fig. 19 (c) and (d) verifies the dynamic improvement using the adaptive step $\triangle$ adp.

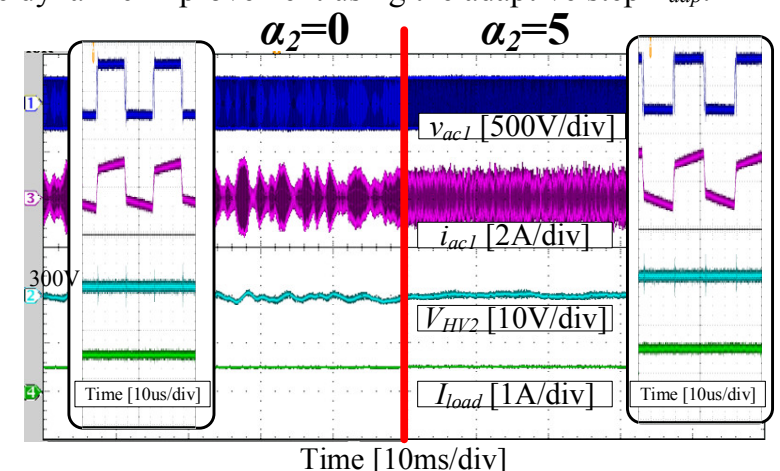

Fig. 18. Sampling noise resistance of the proposed MDCS-MPC.

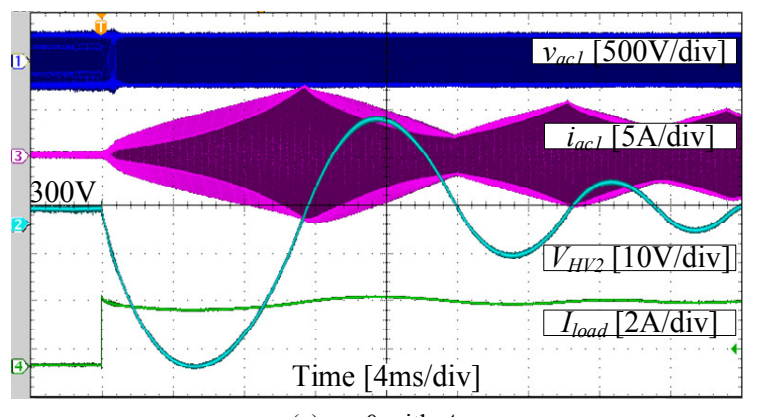

(a) $\alpha_{2}=0$ with $\Delta_{f}$

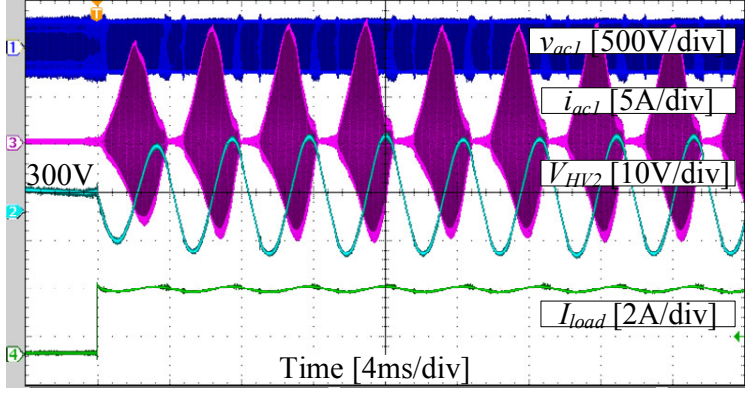

(b) $\alpha_{2}=0$ with $\Delta_{\text {adp }}$ 

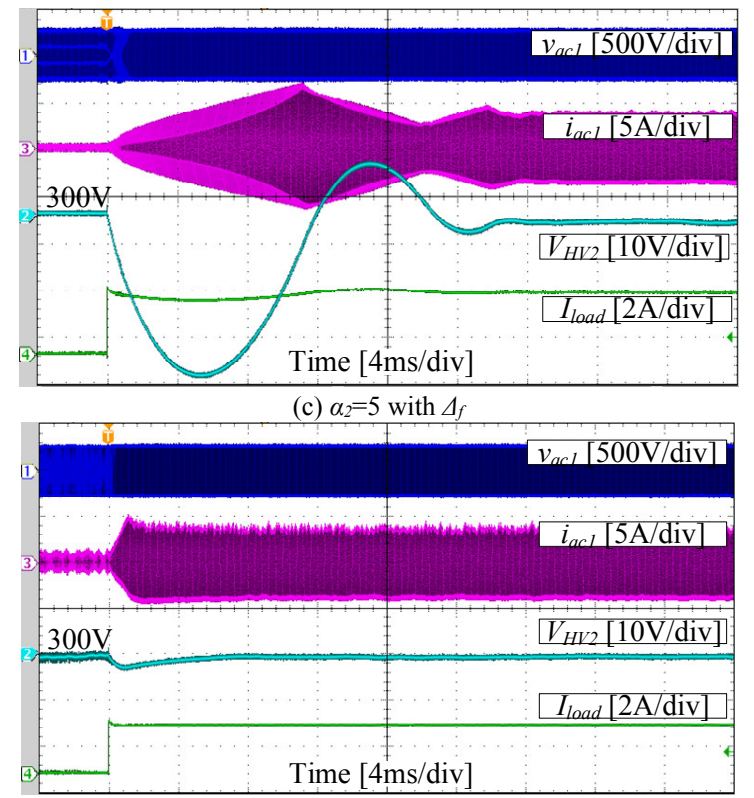

(d) $\alpha_{2}=5$ with $\triangle_{a d p}$

Fig. 19. Verification on effectiveness of $G_{2}$ and adaptive step $\Delta_{a d p}$. (a) $\alpha_{2}=0$ with $\Delta_{f}$; (b) $\alpha_{2}=0$ with $\Delta_{a d p} ;$ (c) $\alpha_{2}=5$ with $\Delta_{f}$; (d) $\alpha_{2}=5$ with $\Delta_{a d p}$

The experiment results with PPLs are provided in Fig. 20 The PI controller is designed and tuned referring to the design approach described in the chapter 6.7 of [36]. The voltage waveforms verify that the proposed MDCS-MPC provides stiff voltage regulation in presence of PPLs while PI controller has limitations on voltage dip and overshoot.
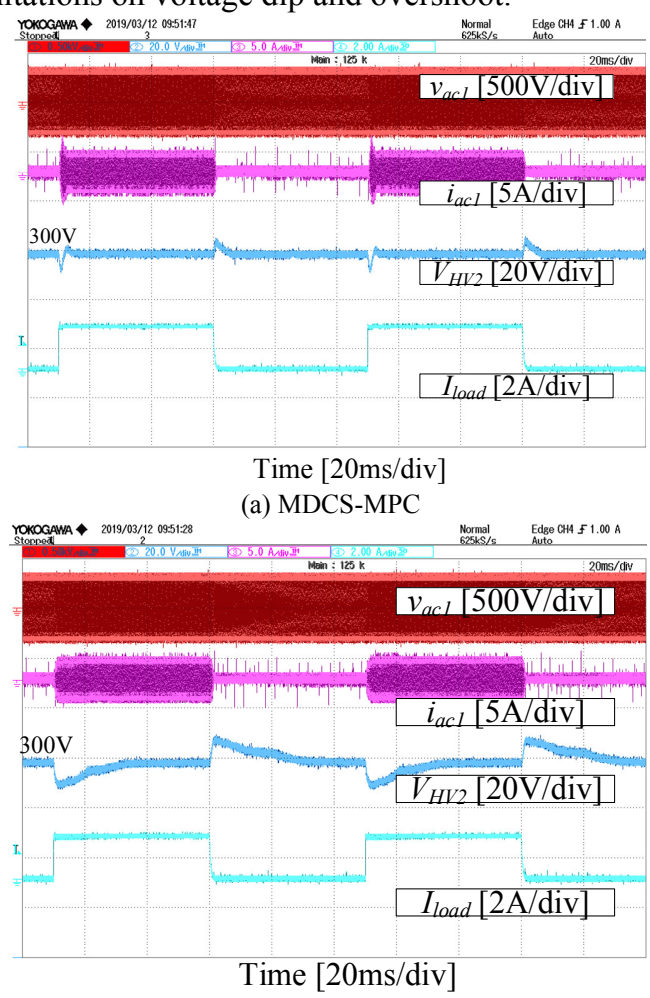

(b) PI control

Fig. 20. Comparison between (a) proposed MDCS-MPC, (b) PI control when the DAB converter is loaded with PPLs@ $20 \mathrm{~Hz}$
Experiments in presence of $L_{s r c}$ have also been conducted for both MDCS-MPC and the PI controller. Results are shown in Fig. 21. DAB regulated with the PI controller is not affected by $L_{s r c}$ when comparing Fig. 20(b) and Fig. 21(b). However, $V_{H V 1}$ in Fig. 21(a) has larger variation compared to Fig. 21(b) due to faster transition of input source current with MDCSMPC. And due to the variation in $V_{H V l}$, the transition with MDCS-MPC is marginally deteriorated as concluded from comparison of Fig. 20(a) and Fig. 21(a).

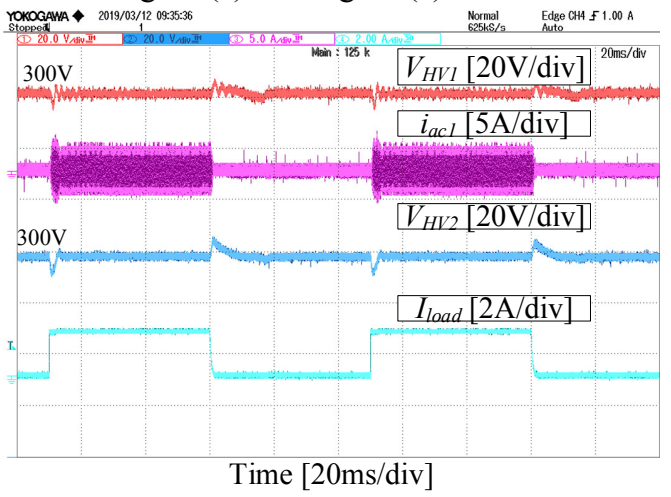

(a) MDCS-MPC

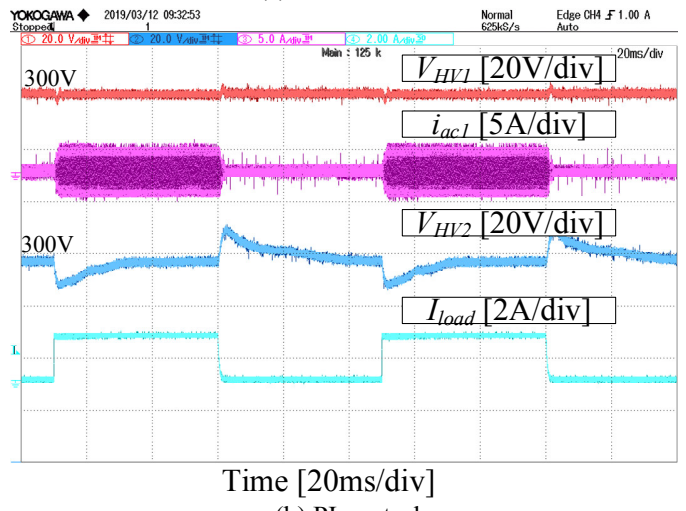

(b) PI control

Fig. 21. Comparison between (a) proposed MDCS-MPC, (b) PI control in presence of $L_{s r c}$ with the DAB loaded with PPLs @20Hz.

\section{CONCLUSIONS}

In this paper, a Moving-Discretized-Control-Set ModelPredictive-Control (MDCS-MPC) is proposed to Dual-ActiveBridge converters supplying Pulsed-Power-Loads (PPLs) in shipboard naval DC micro-grids. The principle of MDCSMPC is intuitively introduced, based on which a cost function is proposed. The cost function, on one hand, provides resonance damping for load voltage; On the other hand, it enhances the sampling noise resistance. The adaptive step is also proposed in this paper for MDCS-MPC. It drastically increases the transition dynamic of the converter.

Performance evaluations on load/source disturbance rejection have also been carried out. The proposed MDCSMPC prevails all other control methods with similar sampling requirements. Experiments are carried out to further verify the salient performance of the MDCS-MPC. MDCS-MPC provides stiff voltage regulation when DAB is loaded with PPLs and in the presence of input voltage disturbance. 


\section{REFERENCES}

[1] E. Skjong, E. Rodskar, M. Molinas, T. A. Johansen, and J. Cunningham, "The Marine Vessel's Electrical Power System: From its Birth to Present Day," Proc. IEEE, vol. 103, no. 12, pp. 24102424, Dec. 2015

[2] E. Skjong, R. Volden, E. Rodskar, M. Molinas, T. A. Johansen, and J. Cunningham, "Past, Present, and Future Challenges of the Marine Vessel's Electrical Power System," IEEE Trans. Transp. Electrif., vol. 2, no. 4, pp. 522-537, Dec. 2016.

[3] M. M. Mardani, M. H. Khooban, A. Masoudian, and T. Dragicevic, "Model Predictive Control of DC-DC Converters to Mitigate the Effects of Pulsed Power Loads in Naval DC Microgrids," IEEE Trans. Ind. Electron., pp. 1-1, 2018.

[4] M. Farhadi and O. Mohammed, "Adaptive Energy Management in Redundant Hybrid DC Microgrid for Pulse Load Mitigation," IEEE Trans. Smart Grid, vol. 6, no. 1, pp. 54-62, Jan. 2015.

[5] S. Jothibasu and S. Santoso, "New Electric Shipboard Topologies for High Resiliency," IEEE Trans. Power Syst., vol. 33, no. 3, pp. 2975-2983, May 2018

[6] M. M. S. Khan, M. O. Faruque, and A. Newaz, "Fuzzy Logic Based Energy Storage Management System for MVDC Power System of All Electric Ship," IEEE Trans. Energy Convers., vol. 32, no. 2, pp. 798-809, Jun. 2017.

[7] M. Farhadi and O. A. Mohammed, "Performance Enhancement of Actively Controlled Hybrid DC Microgrid Incorporating Pulsed Load," IEEE Trans. Ind. Appl., vol. 51, no. 5, pp. 3570-3578, Sep. 2015 .

[8] F. Boattini, J.-P. Burnet, and G. Skawinski, "POPS: The 60MW power converter for the PS accelerator: Control strategy and performances," in 2015 17th European Conference on Power Electronics and Applications (EPE'15 ECCE-Europe), 2015, pp. 110.

[9] L. Chen, L. Tarisciotti, A. Costabeber, Q. Guan, P. Wheeler, and P. Zanchetta, "Phase-Shift-Modulation for a current-fed isolated DCDC converter in More Electric Aircraft," IEEE Trans. Power Electron., pp. 1-1, 2018

[10] L. Chen, L. Tarisciotti, A. Costabeber, F. Gao, P. Wheeler, and P. Zanchetta, "Advanced modulations for a current-fed isolated DCDC converter with wide voltage operating ranges," IEEE J. Emerg. Sel. Top. Power Electron., pp. 1-1, 2018.

[11] B. Zhao, Q. Song, W. Liu, and Y. Sun, "Overview of Dual-ActiveBridge Isolated Bidirectional DC-DC Converter for HighFrequency-Link Power-Conversion System," IEEE Trans. Power Electron., vol. 29, no. 8, pp. 4091-4106, Aug. 2014.

[12] T. Dragicevic, "Model Predictive Control of Power Converters for Robust and Fast Operation of AC Microgrids," IEEE Trans. Power Electron., vol. 33, no. 7, pp. 6304-6317, Jul. 2018.

[13] L. Tarisciotti et al., "Model Predictive Control for Shunt Active Filters With Fixed Switching Frequency," IEEE Trans. Ind. Appl., vol. 53, no. 1, pp. 296-304, Jan. 2017.

[14] L. Tarisciotti, P. Zanchetta, A. Watson, S. Bifaretti, and J. C. Clare, "Modulated Model Predictive Control for a Seven-Level Cascaded H-Bridge Back-to-Back Converter," IEEE Trans. Ind. Electron., vol. 61 , no. 10 , pp. 5375-5383, Oct. 2014.

[15] P. Karamanakos, T. Geyer, and S. Manias, "Direct Voltage Control of DC-DC Boost Converters Using Enumeration-Based Model Predictive Control," IEEE Trans. Power Electron., vol. 29, no. 2, pp. 968-978, Feb. 2014.

[16] B. Wang, V. R. K. Kanamarlapudi, L. Xian, X. Peng, K. T. Tan, and P. L. So, "Model Predictive Voltage Control for Single-Inductor Multiple-Output DC-DC Converter With Reduced Cross Regulation," IEEE Trans. Ind. Electron., vol. 63, no. 7, pp. 41874197, Jul. 2016

[17] F. M. Oettmeier, J. Neely, S. Pekarek, R. DeCarlo, and K. Uthaichana, "MPC of Switching in a Boost Converter Using a Hybrid State Model With a Sliding Mode Observer," IEEE Trans. Ind. Electron., vol. 56, no. 9, pp. 3453-3466, Sep. 2009.

[18] P. Akter, M. Uddin, S. Mekhilef, N. M. L. Tan, and H. Akagi, "Model predictive control of bidirectional isolated DC-DC converter for energy conversion system," Int. J. Electron., vol. 102 , no. 8 , pp. $1407-1427$, Aug. 2015
[19] S. Dutta, S. Hazra, and S. Bhattacharya, "A Digital Predictive Current-Mode Controller for a Single-Phase High-Frequency Transformer-Isolated Dual-Active Bridge DC-to-DC Converter," IEEE Trans. Ind. Electron., vol. 63, no. 9, pp. 5943-5952, Sep. 2016.

[20] S. Dutta, S. Bhattacharya, and M. Chandorkar, "A novel predictive phase shift controller for bidirectional isolated dc to dc converter for high power applications," in 2012 IEEE Energy Conversion Congress and Exposition (ECCE), 2012, pp. 418-423.

[21] Z. Shan, J. Jatskevich, H. H.-C. Iu, and T. Fernando, "Simplified Load-Feedforward Control Design for Dual-Active-Bridge Converters With Current-Mode Modulation," IEEE J. Emerg. Sel. Top. Power Electron., vol. 6, no. 4, pp. 2073-2085, Dec. 2018.

[22] W. Song, N. Hou, and M. Wu, "Virtual Direct Power Control Scheme of Dual Active Bridge DC-DC Converters for Fas Dynamic Response," IEEE Trans. Power Electron., vol. 33, no. 2, pp. 1750-1759, Feb. 2018.

[23] A. R. Alonso, J. Sebastian, D. G. Lamar, M. M. Hernando, and A. Vazquez, "An overall study of a Dual Active Bridge for bidirectional DC/DC conversion," 2010 IEEE Energy Conversion Congress and Exposition. pp. 1129-1135, 2010.

[24] Hua Bai, Chunting Mi, Chongwu Wang, and S. Gargies, "The dynamic model and hybrid phase-shift control of a dual-activebridge converter," in 2008 34th Annual Conference of IEEE Industrial Electronics, 2008, pp. 2840-2845.

[25] K. Zhang, Z. Shan, and J. Jatskevich, "Large- and Small-Signal Average-Value Modeling of Dual-Active-Bridge DC-DC Converter Considering Power Losses," IEEE Trans. Power Electron., vol. 32, no. 3, pp. 1964-1974, Mar. 2017

[26] Hengsi Qin and J. W. Kimball, "Generalized Average Modeling of Dual Active Bridge DC-DC Converter," IEEE Trans. Power Electron., vol. 27, no. 4, pp. 2078-2084, Apr. 2012.

[27] L. Shi, W. Lei, Z. Li, J. Huang, Y. Cui, and Y. Wang, "Bilinear Discrete-Time Modeling and Stability Analysis of the Digitally Controlled Dual Active Bridge Converter," IEEE Trans. Power Electron., vol. 32, no. 11, pp. 8787-8799, Nov. 2017.

[28] K. Shen, J. Feng, and J. Zhang, "Finite Control Set Model Predictive Control with Feedback Correction for Power Converters," China Electrotech. Soc. Trans. Electr. Mach. Syst., vol. 2, no. 3, pp. 312-319, Sep. 2018

[29] T. Dragicevic and M. Novak, "Weighting Factor Design in Model Predictive Control of Power Electronic Converters: An Artificial Neural Network Approach," IEEE Trans. Ind. Electron., pp. 1-1, 2018.

[30] Yanhui Xie, Jing Sun, and J. S. Freudenberg, "Power Flow Characterization of a Bidirectional Galvanically Isolated HighPower DC/DC Converter Over a Wide Operating Range," IEEE Trans. Power Electron., vol. 25, no. 1, pp. 54-66, Jan. 2010.

[31] R. P. Aguilera, P. Lezana, and D. E. Quevedo, "Finite-Control-Set Model Predictive Control With Improved Steady-State Performance," IEEE Trans. Ind. Informatics, vol. 9, no. 2, pp. 658667, May 2013.

[32] G. Du, J. Li, and Z. Liu, "The improved model predictive control based on novel error correction between reference and predicted current," in 2018 IEEE Applied Power Electronics Conference and Exposition (APEC), 2018, pp. 3005-3010.

[33] S. Guenter et al., "Load Control for the DC Electrical Power Distribution System of the More Electric Aircraft," IEEE Trans. Power Electron., pp. 1-1, 2018.

[34] A. Rodriguez, A. Vazquez, D. G. Lamar, M. M. Hernando, and J. Sebastian, "Different Purpose Design Strategies and Techniques to Improve the Performance of a Dual Active Bridge With Phase-Shift Control," IEEE Trans. Power Electron., vol. 30, no. 2, pp. 790-804, Feb. 2015.

[35] T. Dragicevic, "Dynamic Stabilization of DC Microgrids with Predictive Control of Point of Load Converters," IEEE Trans. Power Electron., pp. 1-1, 2018.

[36] K. Johan and T. Hägglund, Advanced PID control. ISA-The Instrumentation, Systems, and Automation Society, 2006. 


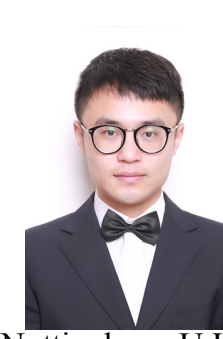

Linglin Chen received his MSc degree in Electrical Engineering from Zhejiang University, Hangzhou, China, in 2016. He was a visiting scholar with the Department of Energy Technology, Aalborg University, Aalborg, Denmark. He is currently with the Power Electronics, Machine \& Control (PEMC) group in the University of Nottingham, U.K. as a Ph.D. candidate.

His current research interests include high power AC/DC converters, high current DC/DC power converters, model predictive control and power system in more electric aircrafts.

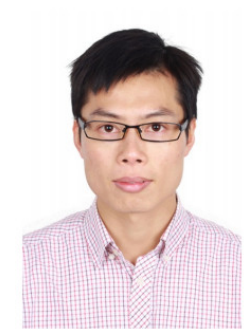

Shuai Shao (M'17) was born in Hunan Province, China, in 1987. He received the B.S. degree in 2010 from Zhejiang University, China, and the $\mathrm{PhD}$ degree in Electrical Engineering from the University of Nottingham, UK, in 2015. In December 2015, he joined the College of Electrical Engineering, Zhejiang University as a lecturer. His research interests include solid state transformers, bidirectional dc-dc converters and fault detection in power converters

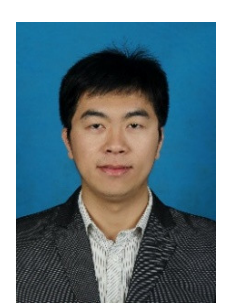

Qian Xiao received the B.S. and M.S. degrees in electrical engineering from Hebei University of Technology, Tianjin, China, in 2011 and 2014 respectively. Since November 2018, he has been a visiting researcher with the Department of Energy Technology, Aalborg University, Aalborg, Denmark.

$\mathrm{He}$ is currently pursuing the Ph.D. degree in electrical engineering, Tianjin University, Tianjin, China. His research interests are multilevel converters, DC/DC converters, and power electronics for distributed generation, micro-grid, and HVDC.

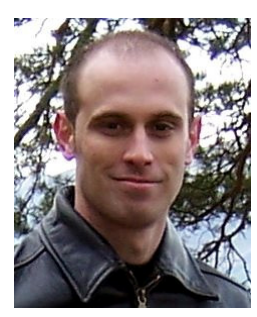

Luca Tarisciotti (S'12-M'15) received the Master degree in Electronic Engineering from the University of Rome "Tor Vergata" in 2009 and his Ph.D. degree in Electrical and Electronic Engineering from the PEMC group, University of Nottingham in 2015. In the same year he became Research Fellow at the University of Nottingham, UK. In 2018, he started working at the University of Chile, Santiago, Chile as adjunct Professor. He is currently working as Assistant Professor at the University Andres Bello, Santiago, Chile. His research interests includes Matrix converters, DC/DC converters, Multi-level converters, Advanced modulation schemes, and Advanced power electronics converter control.

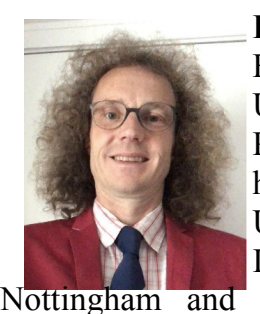

Patrick Wheeler (SM'11) received his BEng [Hons] degree in 1990 from the University of Bristol, UK. He received his $\mathrm{PhD}$ degree in Electrical Engineering for his work on Matrix Converters from the University of Bristol, UK in 1994.

In 1993 he moved to the University of Nottingham and worked as a research assistant in the Department of Electrical and Electronic Engineering. In 1996 he became a Lecturer in the Power Electronics, Machines and Control Group at the University of Nottingham, UK. Since January 2008 he has been a Full Professor in the same research group. He is currently Head of the Department of Electrical and Electronic Engineering at the University of Nottingham and the Li Dak Sum Chair Professor in Electrical and Aerospace Engineering at the University of Nottingham, China. He is a member of the IEEE PELs AdCom and was an IEEE PELs Distinguished Lecturer from 2013 to 2017. He has published 500 academic publications in leading international conferences and journals.

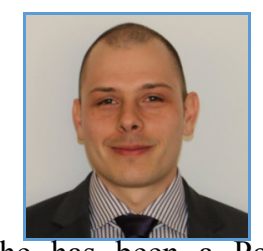

Tomislav Dragičević (S'09-M'13SM'17) received the M.Sc. and the industrial Ph.D. degrees in Electrical Engineering from the Faculty of Electrical Engineering, Zagreb, Croatia, in 2009 and 2013, respectively. From 2013 until 2016 he has been a Postdoctoral research associate at Aalborg University, Denmark. From March 2016 he is an Associate Professor at Aalborg University, Denmark where he leads an Advanced Control Lab.

He made a guest professor stay at Nottingham University, UK during spring/summer of 2018. His principal field of interest is design and control of micro-grids, and application of advanced modeling and control concepts to power electronic systems. $\mathrm{He}$ has authored and co-authored more than 155 technical papers (more than 70 of them are published in international journals, mostly IEEE Transactions) in his domain of interest, 8 book chapters and a book in the field.

He serves as Associate Editor in the IEEE TRANSACTIONS ON INDUSTRIAL ELECTRONICS, in IEEE Emerging and Selected Topics in Power Electronics and in IEEE Industrial Electronics Magazine. Dr. Dragičević is a recipient of the Končar prize for the best industrial $\mathrm{PhD}$ thesis in Croatia, and a Robert Mayer Energy Conservation award. 